\title{
ESTUDIOS ETNOBOTÁNICOS Y FLORÍSTICOS DE LOS BOSQUES AMENAZADOS DE POLYLEPIS INCARUM Y POLYLEPIS PACENSIS (ROSACEAE) EN BOLIVIA
}

\author{
Ethnobotanical and floristic studies of endangered Polylepis incarum and Polylepis pacensis \\ (Rosaceae) forests in Bolivia

\begin{abstract}
ROSEMBER HURTADO ${ }^{1}$, ARELY N. PALABRAL-AGUILERA ${ }^{1}$, ALEJANDRA I. DOMIC ${ }^{1,2}$, M. ISABEL GÓMEZ ${ }^{3}$ \& MÁXIMO LIBERMAN ${ }^{4}$
\end{abstract}

\begin{abstract}
Resumen: Los bosques de Polylepis (Rosaceae) constituyen hábitats importantes para la flora y fauna altoandina y proveen servicios ecosistémicos directos a las comunidades indígenas. Sin embargo, existen pocos relevamientos florísticos así como estudios etnobotánicos en estos ecosistemas boscosos. Evaluamos la composición florística, los usos socioeconómicos de las especies y la valoración local sobre los bosques de $P$. pacensis y $P$. incarum en los Andes occidentales bolivianos. Registramos un total de 46 especies de plantas en los bosques de $P$. pacensis y 58 especies en los bosques de $P$. incarum. Cada uno incluye dos especies endémicas, respectivamente, Aa trilobulata y Calceolaria bartsiifolia, e Hieracium padcayense y Calceolaria bartsiifolia. En total 13 especies medicinales son utilizadas para tratar una variedad de enfermedades. Las comunidades indígenas locales utilizan Polylepis como combustible, medicina, material de construcción y poseen un importante valor ritual. El $54 \%$ de los entrevistados mencionaron que la cobertura de $P$. pacensis se mantuvo durante los últimos 10 años; mientras que el $44 \%$ de los entrevistados consideró que los bosques de $P$. incarum aumentaron y el $40 \%$ consideró que éstos disminuyeron. Se precisan programas de conservación que involucren la participación activa de las comunidades indígenas locales, para mejorar la valoración de estos ecosistemas boscosos.
\end{abstract}

Palabras clave: Bosques altoandinos, composición florística, etnobotánica, Polylepis, valoración local.

Summary: Polylepis forests constitute important habitats for an array of Andean fauna and flora and provide direct ecosystem services to local indigenous communities. However, there are very few floristic surveys as well as ethnobotanic studies in these forest ecosystems. In this study, we evaluated the floristic composition of these forests, the socio-economic use of plant species, and the local assessment of past cover of $P$. pacensis and $P$. incarum forests in the western Bolivian Andes. We recorded a total of 46 plant species in $P$. pacensis forests and 58 species in $P$. incarum forests. They each include two endemic species, respectively, Aa trilobulata and Calceolaria bartsiifolia, and Hieracium padcayense and Calceolaria bartsiifolia. At least 13 species are medicinal and used to treat a variety of illnesses. Local indigenous communities use Polylepis as fuel, medicine, wood, and building material and it possesses an important ritual value. Fifty four percent of the interviewers considered that $P$. pacensis cover stayed unchanged

\footnotetext{
${ }^{1}$ Herbario Nacional de Bolivia, Instituto de Ecología. La Paz, Bolivia. E-mail: rosemberh@gmail.com; arely.palabral@ gmail.com

${ }^{2}$ The Pennsylvania State University, University Park, Estados Unidos. Email: alejandradomic@gmail.com

${ }^{3}$ Colección Boliviana de Fauna, Museo Nacional de Historia Natural. La Paz, Bolivia. E-mail: isabel.gomez.u@gmail. com

${ }^{4}$ Universidad Mayor de San Andrés. La Paz, Bolivia. E-mail: sajamaes@yahoo.es
} 
for the last 10 years; whereas $44 \%$ perceived that $P$. incarum cover has increased, and $40 \%$ considered that it has decreased over the last 10 years. Conservation programs need to include the active participation of local indigenous communities, in order to improve the local valorization of these forest ecosystems.

Key words: Ethnobotany, floristic composition, High Andean forests, local valuation, Polylepis.

\section{Introducción}

Los bosques altoandinos albergan una gran diversidad de especies, incluyendo algunas endémicas y en peligro de extinción. Los bosques de Polylepis son ecosistemas únicos que se distribuyen en laderas escarpadas y roquedales a lo largo de toda la Cordillera Andina (Kessler, 1995; Fjeldså \& Kessler, 1996). Estos bosques constituyen importantes hábitats para la fauna y flora andina. Por ejemplo, en Bolivia, 266 especies de aves utilizan los bosques como áreas de anidamiento, reproducción y alimentación (Fjeldså \& Kessler, 1996; Fjeldså, 2002; Herzog et al., 2002, 2003). Además, existen en Bolivia 537 especies de plantas vasculares presentes en los bosques de Polylepis (Gareca et al., 2010). Sin embargo, las estimaciones de riqueza de plantas están todavía incompletas debido a que no se han realizado relevamientos sistemáticos en los remanentes boscosos de todas las especies del género. Gareca et al. (2010) sugieren que los estimados todavía son bajos y es posible encontrar más especies en estos ecosistemas boscosos bolivianos.

Los bosques de Polylepis también proveen importantes servicios ecosistémicos, de los cuales las comunidades indígenas son beneficiarios directos. Por ejemplo, estos bosques son una importante fuente de leña y madera. Sin embargo, a pesar de que varios estudios resaltan la importancia de estos bosques como fuente de plantas medicinales (Fjeldså \& Kessler, 1996; Kessler, 2002; Gareca et al., 2010), existen muy pocas evaluaciones sistemáticas que hayan caracterizado los usos socioeconómicos de las especies de Polylepis así como la flora asociada.

En Bolivia, existen 13 especies de Polylepis todas ellas amenazadas (MMAyA, 2012). Estas especies se consideran particularmente sensibles a las actividades humanas y la pérdida del hábitat asociado con bajas tasas de regeneración y crecimiento, alto grado de especialización ecológica y reducido tamaño de las poblaciones remanentes (Kessler \& Driesch, 1993; Capriles \& Flores, 2002; Hensen, 2002; Domic \& Capriles, 2009; Domic et al., 2014). Por ejemplo, Polylepis incarum (Bitter) M. Kessler \& SchmidtLeb. se encuentra "en peligro" (Arrázola et al., 2012), aunque se propone que sea categorizado como "en peligro crítico", ya que se estima que los bosques remanentes sólo cubren 45,12 ha, y están restringidos a las orillas del Lago Titicaca en Bolivia (Domic et al., 2017). Otra especie amenazada es P. pacensis M. Kessler \& Schmidt-Leb., cuyos bosques endémicos se restringen a la Cordillera Occidental Andina boliviana y se encuentran altamente degradados por la quema, la tala y la expansión de la agricultura (Arrázola \& Coronado, 2012; Domic et al., 2015).

En este estudio realizamos: (1) un relevamiento florístico, (2) una evaluación de los usos etnobotánicos de Polylepis y la flora asociada, y (3) una evaluación de la valoración de las comunidades indígenas locales sobre la importancia de estos ecosistemas. Nos enfocamos en los bosques de Polylepis incarum y $P$. pacensis porque se encuentran sujetos a una intensa presión antrópica, y la documentación de la flora y los datos etnobotánicos pueden proveer información sobre la importancia ecológica y socioeconómica de estos bosques (Villagrán \& Castro, 2003; Villagrán et al., 2003; Beck et al., 2010). Un entendimiento más profundo de la interacción de los grupos indígenas con los bosques altoandinos puede aportar 
información esencial para enriquecer los programas de conservación que involucren la participación activa de la población local.

\section{Materiales y Métodos}

\section{Área de estudio}

Los bosques de Polylepis pacensis se encuentran distribuidos entre los 3500-3800 $\mathrm{m}$ de altitud en la ladera occidental del nevado Illimani, aproximadamente a $50 \mathrm{~km}$ al sureste de la cuidad de La Paz, Bolivia. La vegetación es característica de la Puna húmeda con influencia del ecosistema de Yungas (Navarro \& Ferreira, 2007). Las comunidades asentadas en las cercanías a los bosques son de origen Aymara y la economía se basa en la agricultura a pequeña escala, caracterizada por el cultivo de verduras y tubérculos y, en menor grado, en la actividad minera. En este estudio evaluamos cuatro fragmentos boscosos de $P$. pacensis ubicados cerca a las comunidades de Cohoni, Cayimbaya, Atahuallani y Pucaya en el departamento de La Paz, al sureste de la ciudad de La Paz.

Los bosques de Polylepis incarum se encuentran distribuidos en los alrededores del Lago Titicaca, entre los 3600-4100 m de altitud. La vegetación es típica de la Puna húmeda y subhúmeda del norte de Bolivia (Navarro et al., 2010). La región se caracteriza por una larga historia de ocupación humana cuyas comunidades de origen Aymara se dedican a la actividad agrícola, especialmente tubérculos andinos, cereales y leguminosas (Vacher et al., 1991), cría de ganado y turismo. Evaluamos cuatro fragmentos boscosos de $P$. incarum ubicados en las comunidades de Villa Molino, Sampaya, Puerto Acosta, Copacati y adicionalmente Pococata, en el departamento de La Paz.

\section{Especies estudiadas}

Polylepis incarum y $P$. pacensis incluyen árboles y arbustos, distribuidos en forma de pequeños fragmentos y como individuos dispersos en laderas y quebradas rocosas. Ambas especies se encuentran categorizadas como "en peligro" para Bolivia (Arrázola
\& Coronado, 2012; Arrázola et al., 2012). Las principales amenazas constituyen la quema, el sobrepastoreo, la expansión de cultivos agrícolas y asentamientos humanos, y la extracción de leña (Martínez \& Villarte, 2009; Arrázola \& Coronado, 2012; Arrázola et al., 2012).

\section{Composición florística}

Para determinar la composición florística de los bosques de $P$. pacensis y $P$. incarum realizamos colectas libres de especies vasculares y helechos durante la época seca en 2014. Muchas gramíneas se encontraban en estado estéril y no fueron coleccionadas por lo que prevemos un sesgo negativo en la riqueza de esa familia. El esfuerzo de muestreo fue de una visita a cada bosque. Las colecciones botánicas fueron identificadas y depositadas en el Herbario Nacional de Bolivia (LPB).

\section{Entrevistas semi-estructuradas}

El estudio etnobotánico fue llevado a cabo en las comunidades adyacentes a los bosques de $P$. incarum y P. pacensis. Para evaluar los usos etnobotánicos y la valoración local sobre los bosques de $P$. pacensis, realizamos entrevistas semi-estructuradas a 36 mujeres y 17 hombres de origen Aymara, entre 16 y 70 años. En el caso de los bosques de $P$. incarum realizamos un total de 43 entrevistas a 25 mujeres y 18 hombres, entre 13 y 75 años, también de origen Aymara. Los entrevistados accedieron libremente a participar, previa explicación sobre la investigación llevada a cabo.

La técnica de entrevista en ambas regiones fue la de "entrevista con planta" en la cual el investigador mostró plantas frescas para que los entrevistados puedan mencionar su nombre común y usos (Alexiades, 1996). Se realizaron seis preguntas: sobre el nombre de la planta, sobre su utilidad y la de sus diferentes partes (hojas, flores, frutos), y sobre la estimación de la cobertura local de los bosques en comparación a diez años atrás.

\section{Análisis de datos}

La información recopilada en las entrevistas fue organizada en categorías de uso, según el método modificado de Paniagua-Zambrana 
et al. (2010). Las categorías de uso fueron: "medicinales", plantas o partes de estas que sirven para curar o aliviar dolencias y enfermedades; "combustible", plantas usadas como leña o carbón; "construcción", plantas usadas para la construcción de viviendas, como postes, listones y corrales; "herramientas", plantas usadas para la elaboración de arados y cucharas, entre otros; "veterinario", plantas usadas para curar enfermedades de animales domésticos; "ambiental", plantas que benefician a otras formas de vida o son empleadas como indicadores ambientales; "tintóreo", plantas usadas para tinción de lana de oveja y tejidos; y "ritual", plantas usadas en ceremonias o rituales de sanación espiritual.

\section{Resultados}

\section{Composición florística}

Se documentó un total de 93 especies de plantas vasculares, pertenecientes a 40 familias (Tabla 1) de las cuales un total de 58 especies se encontraron en los bosques de $P$. incarum. Se registró la presencia de Hieracium padcayense Sleumer y Calceolaria bartsiifolia Wedd., especies endémicas de Bolivia. Registramos un total de 46 especies en los bosques de P. pacensis, incluyendo dos endémicas Aa trilobulata Schltr. y Calceolaria bartsiifolia. Sólo 11 especies se encuentran presentes tanto en los bosques de $P$. incarum como de $P$. pacensis. Las familias con mayor número de especies fueron Asteraceae (19 especies) y Fabaceae ( 8 especies).

Usos etnobotánicos de Polylepis pacensis y $P$. incarum

Los entrevistados reportaron principalmente cuatro categorías de uso para $P$. pacensis: combustible (95\%), medicinal (58\%), construcción (10\%) y ambiental $(2,6 \%)$ (Tabla 2). El $95 \%$ de los comuneros (habitantes de una comunidad rural), utilizan comúnmente los troncos y ramas de $P$. pacensis para cocinar y para producir carbón $(37 \%)$, que es comercializado a herreros para fabricar herramientas agrícolas. La madera también es utilizada para construir corrales y postes.
En cuanto a los usos medicinales, $P$. pacensis es utilizada para curar 16 enfermedades, incluyendo dolor de estómago y cabeza, fiebre y tos, y enfermedades más complejas asociadas con el hígado, la próstata y el reumatismo. Las hojas son utilizadas para preparar una infusión para el dolor de estómago, cabeza, caderas $\mathrm{y}$ espalda y para curar tos, bilis y próstata y calentura (fiebre). Las flores preparadas en infusión sirven para el dolor de estómago. La madera seca y parcialmente quemada se hierve y se mezcla con perejil, hierba buena, "wira wira" (Gnaphalium cheiranthifolium Lam.) y limón para curar la calentura, los huesos rotos, el sobreparto y el resfrío. Las cenizas se ingieren con agua hervida con "k'anapaco" (Sonchus oleraceus L.), "wira wira" y limón para curar la bilis. La corteza se unta con grasa de conejo para formar un parche que es utilizado para curar llagas de la piel.

Respecto de los usos rituales, las hojas y corteza molida son preparadas en una infusión e ingeridas para proteger contra las "maldiciones", curar el "mal de ojo" y afecciones provocadas por el 'k'ari k'ari" (entidad maligna que extrae la grasa abdominal cuando las personas duermen, provocándoles luego la enfermedad y la muerte). Se incluyen estos aspectos dentro de la categoría "ritual" porque las preparaciones y la curación son realizadas por "curanderos" o "yatiris", quienes son los especialistas en aspectos sobrenaturales mediante sus propios procedimientos.

Se reportaron siete categorías de uso para $P$. incarum, de las cuales el uso de mayor mención por los entrevistados fue "combustible" (79\%), seguido por "medicinal" (52\%) y "tintóreo" (24\%) (Tabla 2). Registramos 12 usos dentro de la categoría "medicinal" y dos usos dentro de la categoría "combustible". Las hojas son preparadas en infusión para curar infecciones respiratorias, próstata, gripe, dolor de estómago, dolor menstrual y complicaciones durante el parto. La flor mezclada con otras hierbas aplastadas se coloca en el oído cuando le entra agua. El tronco parcialmente quemado es mezclado con hierbas (no documentadas), como infusión, para curar enfermedades de la próstata. La infusión de la corteza sirve para curar la tos, diarrea infantil, pulmones, vesícula y sobreparto. El tronco de $P$. incarum 
R. Hurtado et al., Estudios etnobotánicos y florísticos de bosques de Polylepis incarum y P. pacensis en Bolivia

Tabla 1. Lista de las especies registradas en los bosques de P. incarum y P. pacensis en Bolivia. El símbolo X* indica especie medicinal y el símbolo $\mathrm{X}^{\mathrm{i}}$ indica especie introducida.

Table 1. List of species recorded in the forests of P. incarum and P. pacensis in Bolivia. The symbol $\mathrm{X} *$ indicates medicinal species and the symbol Xi indicates introduced species.

\begin{tabular}{|c|c|c|c|}
\hline Familia & Especie & P. incarum & P. pacensis \\
\hline \multirow[t]{2}{*}{ Alstroemeriaceae } & Bomarea cf. dulcis (Hook.) Beauverd & $x$ & \\
\hline & Bomarea sp. & & $x$ \\
\hline Anacardiaceae & Schinus microphylla I. M. Johnst. & & $x$ \\
\hline Apiaceae & Azorella multifida (Ruiz \& Pav.) Pers. & & $x$ \\
\hline Apocynaceae & Morfoespecie 1 & $x$ & \\
\hline Araliaceae & Oreopanax macrocephalus Decne. \& Planch. ex Wedd. & & $x$ \\
\hline Aspleniaceae & Asplenium sp. & & $x$ \\
\hline \multirow[t]{20}{*}{ Asteraceae } & Achyrocline sp. & $x$ & $x$ \\
\hline & Achyrocline alata (Kunth) DC. & & $x$ \\
\hline & Baccharis latifolia (Ruiz \& Pav.) Pers. & & $\mathrm{X}^{*}$ \\
\hline & Baccharis papillosa Rusby & & $x$ \\
\hline & Baccharis pentlandii DC. & & $x$ \\
\hline & Baccharis tola Phil. var. incarum (Wedd.) Joch. Müll. & $X^{*}$ & $X^{*}$ \\
\hline & Chersodoma sp. & $x$ & \\
\hline & Chersodoma jodopappa (Sch. Bip.) Cabrera & $x$ & \\
\hline & Chuquiraga jussieui J. F. Gmel. & $x$ & $x$ \\
\hline & Gamochaeta sp. & $\mathrm{X}^{*}$ & \\
\hline & Gnaphalium cheiranthifolium Lam. & & $X^{*}$ \\
\hline & Hieracium padcayense Sleumer & $x$ & \\
\hline & Lucilia sp. & $x$ & \\
\hline & Senecio rufescens DC. & $x$ & \\
\hline & Morfoespecie 1 & $x$ & \\
\hline & Morfoespecie 2 & $x$ & \\
\hline & Morfoespecie 3 & $x$ & \\
\hline & Sonchus oleraceus L. & & $x$ \\
\hline & Stevia sp. 1 & & $x$ \\
\hline & Stevia sp. 2 & $x$ & \\
\hline \multirow[t]{2}{*}{ Berberidaceae } & Berberis boliviana Lechl. & & $x$ \\
\hline & Berberis commutata Eichler & & $x$ \\
\hline \multirow[t]{2}{*}{ Bromeliaceae } & Puya sp. 1 & & $x$ \\
\hline & Puya sp. 2 & $x$ & \\
\hline \multirow[t]{3}{*}{ Cactaceae } & Cumulopuntia boliviana (Salm-Dyck) F. Ritter & $x$ & \\
\hline & Echinopsis sp. & $x$ & \\
\hline & Morfoespecie 1 & $x$ & \\
\hline Campanulaceae & Lobelia sp. & $x$ & \\
\hline Caprifoliaceae & Valeriana sp. & $x$ & \\
\hline Cupressaceae & Cupressus sargentii Jeps. & $X^{i}$ & \\
\hline Cyperaceae & Carex sp. & $x$ & \\
\hline Dryopteridaceae & Polystichum orbiculatum (Desv.) J. Rémy \& Fée & $x$ & \\
\hline Equisetaceae & Equisetum bogotense Kunth & & $\mathrm{X}^{*}$ \\
\hline
\end{tabular}




\begin{tabular}{|c|c|c|c|}
\hline Familia & Especie & P. incarum & P. pacensis \\
\hline Ericaceae & Pernettya prostrata (Cav.) DC. & $x$ & \\
\hline Escalloniaceae & Escallonia resinosa (Ruiz \& Pav.) Pers. & & $x$ \\
\hline \multirow[t]{8}{*}{ Fabaceae } & Adesmia spinosissima Meyen ex Vogel & $x$ & \\
\hline & Astragalus sp. & $x$ & \\
\hline & Lupinus sp. & $x$ & \\
\hline & Lupinus altimontanus C. P. Sm. & & $X^{*}$ \\
\hline & Lupinus microphyllus Desr. & $x$ & \\
\hline & Otholobium pubescens (Poir.) J. W. Grimes & & $x$ \\
\hline & Senna aymara H. S. Irwin \& Barneby & $x$ & \\
\hline & Trifolium amabile Kunth & $x$ & \\
\hline \multirow[t]{3}{*}{ Gramineae } & Cortaderia sp. & & $x$ \\
\hline & Festuca sp. & $x$ & \\
\hline & Jarava ichu Ruiz \& Pav. & $x$ & $x$ \\
\hline Hypericaceae & Hypericum sp. & $x$ & \\
\hline \multirow[t]{2}{*}{ Juncaceae } & Juncus ebracteatus E. Mey. & $x$ & \\
\hline & Luzula excelsa Buchenau & & $x$ \\
\hline \multirow[t]{2}{*}{ Lamiaceae } & Clinopodium bolivianum (Benth.) Kuntze & $\mathrm{X}^{*}$ & $\mathrm{X}^{*}$ \\
\hline & Lepechinia meyenii (Walp.) Epling & $X^{*}$ & $x$ \\
\hline Loranthaceae & Tristerix penduliflorus Kuijt & & $x$ \\
\hline Melastomataceae & Brachyotum microdon (Naudin) Triana & & $x$ \\
\hline Myrtaceae & Eucalyptus globulus Labill. & $x^{i}$ & $X^{i}$ \\
\hline Orchidaceae & Aa trilobulata Schltr. & & $x$ \\
\hline \multirow[t]{2}{*}{ Orobanchaceae } & Agalinis lanceolata (Ruiz \& Pav.) D’Arcy & $x$ & $x$ \\
\hline & Castilleja virgata Dombey ex Wedd. & & $x$ \\
\hline Passifloraceae & Passiflora tripartita (Juss.) Poir. & & $x$ \\
\hline Phytolaccaceae & Phytolacca bogotensis Kunth & & $x$ \\
\hline \multirow[t]{4}{*}{ Plantaginaceae } & Digitalis purpurea L. & $x$ & \\
\hline & Plantago sp. & $x$ & \\
\hline & Plantago lanceolata L. & & $x$ \\
\hline & Plantago sericea Ruiz \& Pav. & $x$ & \\
\hline Polemoniaceae & Cantua buxifolia Juss. ex Lam. & $\mathrm{X}^{*}$ & \\
\hline \multirow[t]{2}{*}{ Polygonaceae } & Muehlenbeckia volcanica (Benth.) Endl. & $x$ & \\
\hline & Rumex acetosella L. & $x$ & $x$ \\
\hline Polypodiaceae & Pleopeltis sp. & $x$ & \\
\hline \multirow[t]{3}{*}{ Pteridaceae } & Adiantum orbignyanum Kuhn & $x$ & \\
\hline & Cheilanthes pruinata Kaulf. & $x$ & \\
\hline & Pellaea ternifolia (Cav.) Link & $x$ & \\
\hline Ranunculaceae & Clematis seemannii Kuntze & & $x$ \\
\hline Rhamnaceae & Colletia spinosissima J. F. Gmel. & $x$ & \\
\hline \multirow[t]{5}{*}{ Rosaceae } & Lachemilla pinnata (Ruiz \& Pav.) Rothm. & $x$ & $x$ \\
\hline & Polylepis incarum (Bitter) M. Kessler \& Schmidt-Leb. & $x$ & \\
\hline & Polylepis pacensis M. Kessler \& Schmidt-Leb. & & $x$ \\
\hline & Rosa rubiginosa L. & & $X^{i}$ \\
\hline & Tetraglochin cristatum (Britton) Rothm. & $\mathrm{x}$ & \\
\hline Rubiaceae & Galium corymbosum Ruiz \& Pav. & $\mathrm{x}$ & \\
\hline
\end{tabular}




\begin{tabular}{|c|c|c|c|}
\hline Familia & Especie & P. incarum & P. pacensis \\
\hline \multirow[t]{2}{*}{ Saxifragaceae } & Ribes sucheziense Jancz. & $x$ & \\
\hline & Saxifraga magellanica Poir. & & $x$ \\
\hline Schoepfiaceae & Quinchamalium chilense Molina & & $x$ \\
\hline \multirow[t]{5}{*}{ Scrophulariaceae } & Bartsia crenoloba Wedd. & $x$ & \\
\hline & Buddleja coriacea J. Rémy & $\mathrm{X}^{*}$ & \\
\hline & Calceolaria bartsiifolia Wedd. & & $X^{*}$ \\
\hline & Calceolaria buchtieniana Kraenzl. & & $X^{*}$ \\
\hline & Calceolaria engleriana Kraenzl. & $x$ & $X^{*}$ \\
\hline TOTAL & & 58 & 46 \\
\hline
\end{tabular}

también es utilizado para curar el sobreparto del ganado vacuno, también se prepara una infusión que se da a tomar a los animales.

El tronco es utilizado como leña, carbón y madera para corrales, artesanías, herramientas y utensilios de cocina. Durante los últimos años, la madera también es utilizada para construir juegos de living y comedor que se comercializan en la turística ciudad de Copacabana. $P$. incarum es utilizado también para quemar incienso en rituales como la mesa de ofrenda a la Pachamama, y es plantado cerca de las viviendas como protección contra los "malos espíritus". Los entrevistados mencionaron el uso "ambiental" de la especie porque los árboles "atraen el agua", producen sombra y aumentan la humedad en el suelo. Las hojas solían utilizarse para teñir lana de oveja de color café-verdoso, que era empleada para tejer "agüayos" (típicos textiles andinos).

\section{Usos etnobotánicos de la flora asociada}

Se registró un total de nueve especies de plantas medicinales presentes en los bosques de $P$. pacensis y seis presentes en los bosques de $P$. incarum (Tabla 1). Estas especies son empleadas para aliviar diversas afecciones, desde pequeñas dolencias externas hasta dolores de postparto, riñones, próstata, estómago, entre otros.

Estimación local de la cantidad de bosque y su valoración

El $54 \%$ de los entrevistados consideraron que actualmente existe la misma cantidad

Tabla 2. Categorías de uso y número de usos mencionados por los entrevistados para dos especies de Polylepis en el Departamento de La Paz, Bolivia.

Table 2. Use categories and number of uses mentioned by the interviewees for two Polylepis species in the Department of La Paz, Bolivia.

\begin{tabular}{|ccccc|}
\hline Categorías de uso & \multicolumn{2}{c}{$P$. pacensis } & \multicolumn{2}{c|}{ P. incarum } \\
Combustible & 94,7 & $\begin{array}{c}\text { Número de } \\
\text { usos }\end{array}$ & \% Entrevistados & Número de usos \\
Medicinal & 58,0 & 3 & 79,3 & 2 \\
Construcción & 10,5 & 2 & 51,7 & 12 \\
Herramientas & 0 & 0 & 3,45 & 1 \\
Tintóreo & 0 & 0 & 3,45 & 1 \\
Ritual & 0 & 0 & 24,14 & 1 \\
Ambiental & 2,63 & 1 & 3,45 & 0 \\
Veterinario & 0 & 0 & 0 & 1 \\
Total Usos & & 22 & 3,45 & 19 \\
\hline
\end{tabular}


de bosques de $P$. pacensis en comparación a diez años atrás, el $36 \%$ de los entrevistados consideraron que los bosques aumentaron, y sólo el $10 \%$ consideran que están desapareciendo. En cambio, la estimación local sobre $P$. incarum muestra que el $44 \%$ de los entrevistados consideraron que los bosques aumentaron en relación a 10 años atrás, el 40\% considera que disminuyeron, y el $16 \%$ que la extensión no cambió (Fig. 1).

En relación a la valoración de los comunarios que viven en cercanías a los bosques de $P$. pacensis, el $16 \%$ considera que la planta no es muy importante, otros entrevistados $(16 \%)$ también mencionaron que las plantas de $P$. pacensis perjudican a los cultivos porque "estorban el establecimiento de una chacra" (la "chacra" es un área de cultivo) y es por ello que "hay que cortarlas y sacarlas desde la raíz", lo que requiere un gran esfuerzo físico; algunos consideran que las raíces resecan el suelo. El restante $(68 \%)$ tienen una valoración positiva ya que conocen los usos de la planta.

Los entrevistados también mencionaron que la presión sobre los bosques de $P$. pacensis disminuyó considerablemente durante los últimos años. En el pasado, la producción de carbón era una práctica muy común, caracterizada por cortar los árboles de mayor tamaño y quemarlos. Esta práctica ha disminuido porque las familias utilizan ahora cocinas a gas o usan como leña el eucalipto y otras plantas que son más fáciles de obtener o que en la actualidad son más abundantes.

La valoración de las comunidades sobre la importancia de los bosques de $P$. incarum está dividida. Algunos entrevistados (24\%) consideran que es una planta con mucha utilidad porque posee múltiples usos, pero otros consideran que es sumamente perjudicial para los cultivos (27\%). E1 49\% restante no manifestaron una valoración positiva ni negativa sobre los bosques. En las comunidades y casas donde la presencia de bosques es relativamente lejana (como en la localidad de Pococata), los entrevistados mencionaron una mayor variedad de usos. Mientras que aquellas comunidades que se encuentran en contacto con los bosques, o tienen abundante cantidad de plantas, son los que poseen valoraciones negativas sobre los bosques nativos.

\section{Discusión}

La flora asociada a los bosques de Polylepis está poco estudiada (FernándezTerrazas \& Stahl, 2002; Antezana et al., 2014). Estos bosques se caracterizan por poseer una diversidad florística relativamente

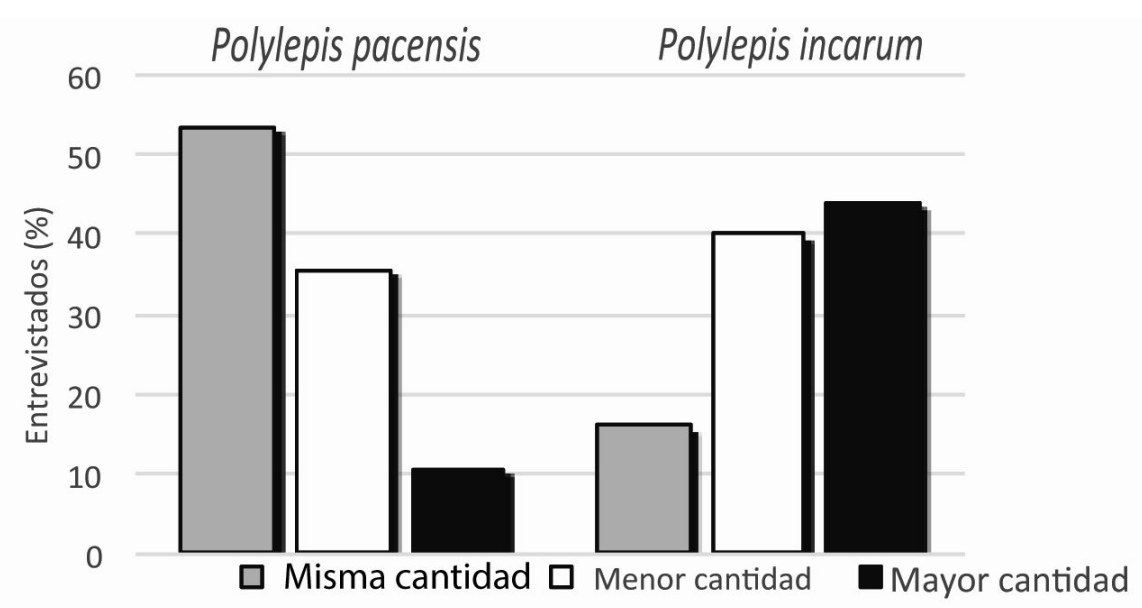

Fig. 1. Estimación local de la cantidad actual de Polylepis en comparación a la existente hace diez años.

Fig. 1. Local estimation of the current amount of Polylepis compared to the existing ten years ago. 
baja, debido a la dominancia arbórea del género y a la elevada altitud en donde se distribuye (Fernández-Terrazas \& Stahl, 2002). Nuestros resultados, al igual que otros estudios florísticos en los bosques de Polylepis en Bolivia, sugieren que estos son relativamente diversos para la Puna Húmeda Altoandina, con una mayor diversidad en el hábito herbáceo y arbustivo. En este estudio reportamos un total de 46 especies correspondientes a 28 familias en los bosques de $P$. pacensis y 58 especies pertenecientes a 27 familias en los bosques de $P$. incarum. Comparaciones con otros estudios en bosques de Polylepis muestran números menores. Por ejemplo, FernándezTerrazas \& Stahl (2002) registraron 16-39 especies de plantas vasculares en los bosques de P. subtusalbida (Bitter) M. Kessler \& Schmidt-Leb., de 20-31 en bosques de $P$. tomentella Wedd. subsp. nana M. Kessler, 19-43 en bosques de P. lanata (Kuntze) M. Kessler \& Schmidt-Leb., y 25-34 en bosques de $P$. tomentella subsp. incanoides $\mathrm{M}$. Kessler. Para el caso de bosques de P. besseri Hieron., Mercado (1998) registró entre 20-40 especies, Hensen (1994) documentó 14-47 especies y Fernández et al. (2001) 29 especies. El estudio de Montesinos-Tubée et al. (2015) realizado con $P$. incarum en el distrito de Lampa, Perú, registra un total de 83 especies pertenecientes a 35 familias, un número superior al registrado en nuestro estudio. Sin embargo, si consideramos el esfuerzo de muestreo, es posible que varias especies, principalmente gramíneas, no fueran registradas por su carácter estéril al momento de realizar las colectas, por lo que recomendamos inventarios más intensivos para completar la lista de especies que presentamos en este estudio.

Varias investigaciones han reportado una vegetación altamente degradada en la región del Lago Titicaca, debido a la larga historia de ocupación humana (Navarro, 2002; Martínez \& Villarte, 2009; Arrázola et al., 2012). La vegetación nativa reportada para los bosques de $P$. incarum se desarrolla mayormente en medio de una matriz de zonas de cultivo y barbechos en diferentes estadios de sucesión. Por otro lado, la presencia de Buddleja coriacea J. Rémy claramente asociada a los cercos vivos para delimitar los cultivos o a las casas de los comunarios.

Varias especies registradas en este estudio sólo se encontraban en un bosque, como es el caso de Ribes sucheziense Jancz., Lupinus microphyllus Desr. o Rosa rubiginosa L. En contraste, otras especies son comunes y de amplia distribución, como ser Lachemilla pinnata (Ruiz \& Pav.) Rothm., Clinopodium bolivianum (Benth.) Kuntze y Lepechinia meyenii (Walp.) Epling, que fueron reportadas en todos los bosques.

La composición florística de los bosques de $P$. pacensis mostró una influencia del bosque yungueño, caracterizado por la presencia de Oreopanax macrocephalus Decne. \& Planch. ex Wedd., Brachyotum microdon (Naudin) Triana y Phytolacca bogotensis Kunth. En las quebradas inaccesibles por la marcada pendiente, y por ende no aptas para cultivos, se encontraron especies nativas como Escallonia resinosa (Ruiz \& Pav.) Pers., Puya sp., Schinus microphylla I. M. Johnst. y Azorella multifida (Ruiz \& Pav.) Pers., especies que prácticamente han desaparecido de otras zonas de la región. También registramos especies parásitas (Quinchamalium chilense Molina) y hemiparásitas (Tristerix penduliflorus Kuijt), la primera suele afectar a las raíces de los Baccharis y otros arbustos (García, 2015), y ha sido encontrada en bosques que han sufrido quemas, lo cual estaría afectando en el vigor de los individuos parasitados. La presencia de especies endémicas de Bolivia como Aa trilobulata y Calceolaria bartsiifolia en los bosques de $P$. pacensis y Hieracium padcayense y Calceolaria bartsiifolia en los bosques de $P$. incarum aumenta el valor de conservación de estos bosques, ya que todavía constituyen el hábitat de especies endémicas.

En relación a la evaluación etnobotánica, documentamos múltiples usos medicinales para $P$. pacensis y $P$. incarum. Ambas especies poseen propiedades medicinales y son utilizadas para curar una diversidad de enfermedades, desde muy leves hasta agudas. Muchos de los usos que registramos no fueron documentados previamente para Polylepis. Ambas especies son utilizadas para curar el 
sobreparto y enfermedades de la próstata. En el caso particular de $P$. pacensis, registramos por primera vez el uso de la especie para tratar los huesos rotos y las heridas en la piel.

Al igual que otras especies del género, $P$. pacensis y $P$. incarum son comúnmente utilizadas para tratar enfermedades de vías respiratorias, hipertensión, tos y reumatismo (De Lucca \& Zalles, 1992; Oblitas, 1992; Sagaseta de Ilurdoz Uranga, 1996; Villagrán \& Castro, 2003; Daud et al., 2008). Sin embargo, se destaca que los entrevistados no mencionaron utilizar ninguna de las especies para curar enfermedades de los riñones, uno de los usos más ampliamente reportados para Polylepis (Oblitas, 1992; Villagrán \& Castro, 2003).

Muchos estudios sobre el uso de Polylepis resaltan las propiedades medicinales del tronco, corteza y hojas (Oblitas, 1992; Villagrán et al., 2003; Villagrán \& Castro, 2003). Existen estudios que muestran que la corteza y ramas de Polylepis poseen propiedades antiinflamatorias (Neto et al., 2000) y que las hojas poseen actividad antimicrobiana e insecticida (Loayza et al., 2002; Bussmann et al., 2010). En este estudio reportamos por primera vez el empleo de las raíces de $P$. incarum y de las flores de $P$. pacensis, lo cual sugiere que podrían tener propiedades citotóxicas pero es necesario llevar a cabo futuros estudios en laboratorio que caractericen los aceites esenciales y evalúen las propiedades antibacterianas.

Ambas especies de Polylepis son utilizadas para curar diversas enfermedades. En algunos casos, los informantes mencionaron utilizar exclusivamente $P$. incarum o $P$. pacensis para preparar las medicinas. En otros casos, Polylepis es combinada con varias especies de plantas para potenciar su función (Hensen, 1992). En este estudio no pudimos registrar de forma detallada todas las plantas empleadas en la medicina tradicional, por lo que consideramos importante realizar estudios más detallados.

Los entrevistados reportaron el uso de hojas de $P$. incarum para la tinción de lana, también documentado para P. racemosa Ruiz \& Pav. en la sierra peruana (Ayma Romay, 2011). Sin embargo, este uso se perdió en las comunidades del Lago Titicaca y fue reemplazado por tintes sintéticos. La disminución del uso de tintes naturales en base a plantas nativas es un fenómeno común en la región Andina debido a la facilidad de la aplicación y obtención de tintes artificiales, así como la falta de transmisión del conocimiento hacia las nuevas generaciones (Pazos, 2017). Durante los últimos años ha surgido el interés de recuperar el uso de tintes naturales y algunas comunidades indígenas han recuperado la tecnología del teñido; en este sentido, el rescate del conocimiento y técnicas ancestrales de textiles constituye uno de los primeros pasos para facilitar la transmisión del conocimiento tradicional.

También resaltamos la importancia ritual de ambas especies de Polylepis. En el caso de $P$. pacensis, ésta es utilizada como protección contra el "mal de ojo" y las afecciones provocadas por el “k'ari k'ari" (Oblitas, 1992). Mientras que $P$. incarum es plantada cerca de las casas como protección contra los "malos espíritus". Estos resultados resaltan la importancia simbólica y ritual de Polylepis como protección contra entidades sobrenaturales (Capriles \& Flores, 2002).

La evaluación también mostró que ambas especies de Polylepis continúan siendo utilizadas como fuente de leña para cocción de alimentos. A pesar de que durante los últimos años el acceso al querosene y el gas licuado de petróleo (GLP) ha aumentado en el área rural de Bolivia, la leña todavía continúa siendo el principal combustible utilizado para cocinar (Mayorga \& Tapia, 2006; Ekouevi \& Tuntivate, 2012), debido principalmente al elevado costo y difícil acceso a GLP (Müller et al., 2014). Tomando en cuenta el reducido tamaño de las poblaciones remanentes de $P$. incarum y $P$. pacensis, consideramos que la extracción de leña todavía constituye una importante amenaza y sugerimos la implementación de medidas que ayuden a reducir la demanda de leña (e.g., estufas ahorradoras de energía, programas de manejo sostenible de los bosques nativos y reforestación).

El uso de madera de $P$. incarum para la elaboración de artesanías y muebles, parece ser una actividad llevada a cabo por pocas familias en los alrededores del Lago Titicaca. Durante la evaluación encontramos que algunos restaurantes de la población de 
Copacabana poseían muebles hechos con madera de la especie. La extracción de madera para este fin constituye una potencial amenaza, en especial si la demanda por muebles se incrementa en el futuro. Es por esto que consideramos importante la implementación de medidas regulatorias a nivel local y nacional.

El principal uso ambiental de Polylepis mencionado por los entrevistados es que "atrae el agua", es decir, que genera humedad en el suelo. La importancia ecosistémica de los bosques de Polylepis es el de albergar una alta biodiversidad de flora altoandina (Gareca et al., 2010; Domic et al., 2015), y de fauna, especialmente aves endémicas y amenazadas (Fjeldså, 2002; Herzog et al., 2002, 2003). Además, los bosques regulan el microclima (Domic et al., 2015), previenen la erosión de los suelos al interceptar el agua en la vegetación y ayudan a la protección y recarga de acuíferos en las cabeceras de los valles (Fjeldså \& Kessler, 1996).

Las valoraciones sobre la importancia de los bosques de $P$. pacensis y $P$. incarum por parte de los entrevistados está dividida y básicamente pocos entrevistados reconocieron los beneficios ecosistémicos que ofrecen. Algunos de los entrevistados mostraron una valoración positiva hacia Polylepis asociado a su valor económico (medicinal, combustible) y ambiental (ej. protección de suelos, producción de oxígeno). Sin embargo, otros entrevistados mostraron actitudes negativas hacia la presencia de los bosques, considerándolos perjudiciales porque absorben la humedad del suelo, debilitan el crecimiento de los cultivos, quitan el espacio a especies forrajeras y quitarlas de los cultivos requiere de un gran esfuerzo físico.

Los seres humanos difieren no sólo en las actividades diarias y en las peculiaridades de la cultura material donde desarrollan su vida; también difieren en la valoración o apreciación de lo que les rodea. La percepción que cada individuo tenga del ambiente depende de su cultura, actividad y rol en éste (Trillo et al., 2016), entre otros aspectos. De esta manera, al ser tan variable el criterio de una persona sobre un determinado elemento de su entorno, es comprensible que exista una disonante división de opiniones. Trillo et al. (2016) ha reportado similar división de opiniones sobre el bosque, donde algunos entrevistados expresaron una valoración positiva y otros una negativa. La valoración positiva de las comunidades sobre los bosques podría deberse a que éstos son espacios para resolver sus expectativas de múltiples propósitos económicos y emocionales (Karppinen, 1998; Majumdar et al., 2008). Por otro lado, es posible que la valoración negativa se deba a que estos bosques no cumplan con las expectativas de los comuneros.

En este estudio registramos 13 especies de plantas medicinales, una cifra relativamente baja en comparación al estudio etnobotánico en la comunidad de Charojo, ubicada en el Departamento de Cochabamba, Bolivia, donde se registraron 111 especies de plantas medicinales presentes en los bosques de $P$. besseri y áreas circundantes (Hensen, 1992). Consideramos que los usos etnobotánicos dentro de los bosques es un tema que debe ser tomado en profundidad en posteriores estudios.

\section{Conclusiones}

Los bosquesde Polylepispacensisy P.incarum albergan numerosas especies de plantas nativas, incluyendo especies endémicas y medicinales. Los indígenas Aymaras se benefician de múltiples maneras del uso y explotación de estos bosques. De forma directa, Polylepis es utilizada como medicina, ritual, combustible y material de construcción e indirectamente para la protección de suelos y hábitat de plantas medicinales. Sin embargo, estos ecosistemas se encuentran amenazados y su pérdida y degradación podría reducir la disponibilidad de plantas socio-económicamente importantes, así como la pérdida de los servicios ambientales que proveen. Resaltamos la necesidad de implementar programas de conservación y manejo sostenible que promuevan la recuperación de los bosques remanentes y mejoren la calidad del hábitat. Asimismo consideramos importante la participación activa de las comunidades indígenas locales, con el fin de mejorar la valoración local sobre la importancia de estos bosques y la implementación de programas de educación y sensibilización ambiental que revaloricen los bosques nativos. 


\section{Agradecimientos}

Agradecemos a las comunidades de Copacati, Puerto Acosta, Sampaya, Villa Molino, Collaracana, Pococata, Pucaya, Atahuallani, Cohoni y Cayimbaya por permitirnos trabajar en su bosque y por brindarnos la información sobre usos de plantas y participar en las entrevistas. A Laura Moya y Elvia Moya por su colaboración en la etapa de entrevistas etnobotánicas. A Rosa Isela Meneses, Cecilia López, Cecilia Vega, Juan Carlos Bermejo, Viviana Urrelo, Loly Vargas, Julieta Acho, Ángela Terán, Nivardo Urzagasti y Adriana Rico por su apoyo en diversas etapas del proyecto. A Alfredo Fuentes, Iván Jiménez, Ariel Lliully y Dora Ibañez por su colaboración en la identificación de especies. El estudio fue financiado por la Agencia Sueca de Desarrollo Internacional (ASDI) en convenio con la Universidad Mayor de San Andrés (UMSA) en el marco del proyecto "Ecología y conservación de dos especies de árboles andinos en peligro de extinción (Polylepis incarum y Polylepis pacensis, Rosaceae) en el departamento de La Paz" y cuenta con el permiso de la Dirección General de Biodiversidad, del Ministerio de Medio Ambiente y Agua.

\section{Bibliografía}

ALEXIADES, M. N. 1996. Collecting ethnobotanical data: an introduction to basic concepts and techniques. En ALEXIADES, M. N. (ed.), Guidelines for ethnobotanical field collectors, pp. 53-94. The New York Botanical Garden, New York.

ANTEZANA, C., M. MERCADO, S. ARRÁZOLA, M. FERNÁNDEZ, B. SOTO \& O. RUIZ. 2014. Guía de plantas de los bosques de Polylepis (kewiña, keñoa) y sus alrededores en el Departamento de Cochabamba Bolivia. Centro de Biodiversidad y Genética, UMSS, Cochabamba. 204 pp.

ARRÁZOLA, S. \& I. CORONADO. 2012. Polylepis pacensis. En LIBRO ROJO de la Flora Amenazada de Bolivia. Vol. I. Zona Andina, La Paz, pp. 338-339.

ARRÁZOLA, S., I. CORONADO, L. TORRICO, R. I. MENESES, G. NAVARRO \& W. FERREIRA. 2012. Polylepis incarum. En LIBRO ROJO de la Flora Amenazada de Bolivia. Vol. I. Zona Andina. La Paz, pp. 44-45.

AYMA ROMAY, A. I. 2011. Beneficios y maleficios de los árboles para los campesinos y su rol en el arreglo de sistemas agroforestales tradicionales en el norte de independencia, Bolivia. Acta Nova 5: 225-246.
BECK, ST. G., A. DOMIC, C. GARCÍA, R. I. MENESES, K. YAGER \& S. HALLOY. 2010. El Parque Nacional Sajama y sus plantas. Departamento de Oruro, Bolivia. Herbario Nacional de Bolivia, La Paz. 250 pp.

BUSSMANN, R. W., G. MALCA, A. GLENN, D. SHARON, B. NILSEN, B. PARRIS, D. DUBOSE, D. RUIZ, J. SALEDA, M. MARTINEZ, L. CARILLO, K. WALKER, A. KUHLMAN \& A. TOWNESMITH. 2010. Toxicity of medicinal plants used in traditional medicine in Northern Peru. J. Ethnopharmacol. 137: 121-140. https://doi.org/10.1016/j.jep.2011.04.071.

CAPRILES, J. M. \& E. FLORES. 2002. The economic symbolic and social importance of the "keñua" (Polylepis spp.) during prehispanic times in the Andean highlands of Bolivia. Ecotropica 8: 225-231.

DAUD, A., N. HABID \& A. SÁNCHEZ. 2008. Actividad antimicrobiana de extractos alcohólicos de hojas y corteza de Polylepis australis Bitter (queñoa). Rev. Cubana Plant. Med. 13: 1-11.

DE LUCCA, M. \& J. ZALLES. 1992. Flora medicinal boliviana, diccionario enciclopédico. SEAPAS. Editorial Los Amigos del Libro, La Paz. 498 pp.

DOMIC, A. I. \& J. M. CAPRILES. 2009. Allometry and effects of extreme elevation on growth velocity of the Andean tree Polylepis tarapacana Philippi (Rosaceae). Plant Ecol. 205: 223-234. https://doi. org/10.1007/s11258-009-9612-5.

DOMIC, A. I., G. CAMILO \& J. M. CAPRILES. 2014. Small-scale farming and grazing reduce regeneration of Polylepis tomentella (Rosaceae) in the semiarid Andes of Bolivia. Biotropica 46: 106-113. https://doi. org/10.1111/btp. 12075.

DOMIC, A. I., M. I. GÓMEZ, R. HURTADO, A. N. PALABRAL-AGUILERA, A. RICO \& M. LIBERMAN. 2015. Los bosques de queñua (Polylepis pacensis) del Valle de La Paz. En MOYA, M. I., R. I. MENESES \& J. SARMIENTO (eds.), Historia Natural de un Valle en Los Andes: La Paz, pp. 206210. Segunda Edición. Museo Nacional de Historia Natural, La Paz, Bolivia.

DOMIC, A. I., A. N. PALABRAL-AGUILERA, M. I. GÓMEZ, R. HURTADO, N. ORTUÑO \& M. LIBERMAN. 2017. Polylepis incarum (Rosaceae) una especie En Peligro Crítico en Bolivia: Propuesta de reclasificación en base al área de ocupación y estructura poblacional. Ecol. Bol. 52: 116-131.

EKOUEVI, K. \& V. TUNTIVATE. 2012. Household energy access for cooking and heating. Lessons learned and the way forward. The World Bank, Washington DC, 52 pp. https://doi.org/10.1596/9780-8213-9604-9.

FERNÁNDEZ, M., M. MERCADO, S. ARRÁZOLA \& E. MARTÍNEZ. 2001. Estructura y composición florística de un fragmento boscoso de Polylepis besseri Hieron subsp. besseri en Sacha Loma (Cochabamba). Rev. Bol. de Ecol. 9: 15-27. 
FERNÁNDEZ-TERRAZAS, E. \& B. STAHL. 2002. Diversity and phytogeography of the vascular flora of the Polylepis forests of the Cordillera de Cochabamba, Bolivia. Ecotropica 8: 163-182.

FJELDSA, J. 2002. Keys areas for conserving the avifauna of Polylepis forest. Ecotropica 8: 125 131.

FJELDSÅ, J. \& M. KESSLER. 1996. Conserving the biological diversity of Polylepis woodlands of the highland of Peru and Bolivia. A contribution to sustainable natural resource management in the Andes. NORDECO, Copenhagen, $250 \mathrm{pp}$.

GARCÍA, E. 2015. Formas de crecimiento y adaptaciones de grupos especiales de plantas en el valle de La Paz. En MOYA, M. I., R. I. MENESES \& J. SARMIENTO (eds.), Historia natural de un valle en los Andes: La Paz, pp. 159-172. Museo Nacional de Historia Natural, La Paz.

GARECA, E., M. HERMY, J. FJELDSA \& O. HONNAY. 2010. Polylepis woodland remnants as biodiversity islands in the Bolivian high Andes. Biodivers. Conserv. 19: 3327-3346. https://doi. org/10.1007/s10531-010-9895-9.

HENSEN, I. 1992. La flora de la comunidad de Chorojo. Su uso, taxonomía científica y vernacular. Serie Técnica 28. Agroecologia Universidad Cochabamba (AGRUCO). Cochabamba, 40 pp.

HENSEN, I. 1994. Estudios ecológicos y fenológicos sobre Polylepis besseri Hieron. en la Cordillera Oriental Boliviana. Ecol. Bol. 23: 21-32.

HENSEN, I. 2002. Impacts of anthropogenic activity on the vegetation of Polylepis woodlands in the region of Cochabamba, Bolivia. Ecotropica 8: 183-203.

HERZOG, S. K., R. SORIA, A. TRONCOSO \& E. MATTHYSEN. 2002. Composition and structure of avian mixed species flocks in a high-Andean Polylepis forest in Bolivia. Ecotropica, 8: 133-143.

HERZOG, S. K., R. A. SORIA \& E. MATTHYSEN. 2003. Seasonal variation in avian community composition in a high-Andean Polylepis (Rosaceae) forest fragment. The Wilson Bull. 115: 438-447. https://doi.org/10.1676/03-048.

KARPPINEN, H. 1998. Values and objectives of nonindustrial private forest owners in Finland. Silva Fenn. 32: 43-59. https://doi.org/10.14214/sf.699.

KESSLER, M. 1995. The genus Polylepis (Rosaceae) in Bolivia. Candollea 50: 131-171.

KESSLER, M. 2002. The "Polylepis problem": where do we stand? Ecotropica 8: 97-110.

KESSLER, M. \& P. DRIESCH. 1993. Causas e historia de la destrucción de bosques altoandinos en Bolivia. Ecol. Bol. 21: 1-18.

LOAYZA, I., L. A. VILASECA, D. LORENZO \& E. DELLACASSA. 2002. Characterization of the essential oil and extracts from the aerial parts of kehuiña (Polylepis besseri). Ecotropica 8: 233-238.
MAJUMDAR, I., L. TEETER \& B. BUTLER. 2008. Characterizing family forest owners: a cluster analysis approach. For. Sci. 54: 176-184.

MARTÍNEZ, O. \& F. VILLARTE. 2009. Estructura dasométrica de las plantas de un parche de Polylepis besseri incarum y avifauna asociada en la Isla del Sol (Lago Titicaca, La Paz - Bolivia). Ecol. Bol. 44: 36-49.

MAYORGA, E. \& A. TAPIA. 2006. The gas era. En FRETES-CIBILS, V., M. GIUGALE \& C. LUFF (eds.), Bolivia. Public policy options for the well-being of all, pp. 163-183. The World Bank, Washington.

MERCADO, M. U. 1998. Vegetación de la ceja de monte yungueña en el Parque Nacional Carrasco (Cochabamba-Bolivia). Rev. Bol. de Ecol. 4: 55-75.

MMAyA. 2012. MINISTERIO DE MEDIO AMBIENTE Y AGUA. LIBRO ROJO de la Flora Amenazada de Bolivia. Vol. I. Zona Andina. La Paz. 600 pp.

MONTESINOS-TUBÉE D. B., W. GALIANO, D. F. BELTRÁN \& A. C. PINTO. 2015. Vegetación de un bosque de Polylepis incarum (Rosaceae) en el distrito de Lampa, Puno, Perú. Rev. Peru. Biol. 22: 87-96. https://doi.org/10.15381/rpb.v22i1.11125.

MÜllER, R., T. PISTORIUS, S. ROHDE, G. GEROLD \& P. PACHECO. 2014. El contexto de la deforestación y degradación de los bosques en Bolivia: causas, actores e instituciones. Documentos Ocasionales CIFOR 100, Bogor. 89 pp.

NAVARRO, G. 2002. Vegetación y unidades biogeográficas. En NAVARRO, G. \& M. MALDONADO (eds.), Geografía Ecológica de Bolivia. Vegetación y Ambientes Acuáticos, pp. 1-500. Centro Ecología Simón I. Patiño Departamento de Difusión, Cochabamba.

NAVARRO, G. \& W. FERREIRA. 2007. Mapa de Vegetación de Bolivia, Esc. 1:250.000. The Nature Conservancy (TNC). Edición digital. Santa Cruz de la Sierra, Bolivia.

NAVARRO, G., S. ARRÁZOLA, J. A. BALDERRAMA, W. FERREIRA, N. DE LA BARRA, C. ANTEZANA, I. GÓMEZ \& M. MERCADO. 2010. Diagnóstico del estado de conservación y caracterización de los bosques de Polylepis en Bolivia y su avifauna. Rev. Bol. de Ecol. 28: 1-35.

NETO, C. C., A. J. VAISBERG, B. N. ZHOU \& D. G. I. KINGSTON. 2000. Cytotoxic triterpene acids from the Peruvian medicinal plant Polylepis racemosa. Planta Med. 66: 483-484. https://doi. org/10.1055/s-2000-8583.

OBLITAS, E. P. 1992. Plantas medicinales de Bolivia. Farmacopea Callawaya. Ed. Los Amigos del Libro, La Paz. 529 pp.

PAZOS, S. 2017. Teñido en base a tintes naturales. Conocimiento y técnicas ancestrales de artistas textiles de Perú y Bolivia. Soluciones Prácticas, Lima, 80 pp. 
PANIAGUA-ZAMBRANA， N., M. J. MACÍA \& R. CÁMARA-LERET. 2010. Toma de datos etnobotánicos de palmeras y variables socioeconómicas en comunidades rurales. Ecol. Bol. 45: 45-68.

SAGASETA DE ILURDOZ URANGA, J. L. 1996. Jampi makikunanchiqpi Kasan. La medicina está en nuestras manos. Proyecto Salud Tiraque. Ediciones Gráficas E. G., Cochabamba, 400 p.

TRILlO, C., B. ARIAS TOLEDO \& S. E. COLANTONIO. 2016. Uso y percepción del bosque por pobladores de diferente tradición cultural de la Laguna de Mar Chiquita, Córdoba, Argentina. Ecol. Austral 26: 7-16.
VACHER, J. J., E. BRASIER DE THUY \& M. LIBERMAN. 1991. Influencia del lago en la agricultura litoral. En DEJOUX C. \& A. ILTIS (eds.), El Lago Titicaca. Síntesis del conocimiento limnológico actual, pp 517530. ORSTOM - HISBOL, La Paz.

VILLAGRÁN, C. \& V. CASTRO. 2003. Ciencia indígena de los Andes del norte de Chile. Editorial Universitaria, Santiago, Chile. 362 pp.

VILlAGRÁN, C., M. ROMO \& V. CASTRO. 2003. Etnobotánica del sur de Los Andes de la primera región de Chile: un enlace entre las culturas altiplánicas y las de quebradas altas del Loa Superior. Chungara, Rev. Antropol. Chilena 35: 73-124. https://doi.org/10.4067/ S0717-73562003000100005.

Original recibido el 9 de marzo de 2018; aceptado el 5 de septiembre de 2018. 